\title{
Thymidylate limitation potentiates cephalosporin activity towards enterococci via an exopolysaccharide-based mechanism
}

\author{
Jessica S. Hoff and Christopher J. Kristich ${ }^{*}$ \\ Department of Microbiology and Molecular Genetics, Medical College of Wisconsin, Milwaukee, \\ WI, USA
}

\begin{abstract}
Multi-drug resistant enterococci are major causes of nosocomial infections. Prior therapy with cephalosporins increases the risk of developing an enterococcal infection due to the intrinsic resistance of enterococci to these antibiotics. While progress has been made towards understanding the genetic and biochemical mechanisms of cephalosporin resistance, available data indicate that as-yet-unidentified resistance factors must exist. Here we describe results of a screen to identify small molecules capable of sensitizing enterococci to broad-spectrum cephalosporins. We found that both Enterococcus faecalis and Enterococcus faecium were sensitized to broad and expanded-spectrum cephalosporins when thymidylate production was impaired, whether by direct inhibition of thymidylate synthase, or by limiting production of cofactors required for its activity. Cephalosporin potentiation is the result of altered exopolysaccharide production due to reduced dTDP-glucose synthesis. Hence, exopolysaccharide production is a previously undescribed contributor to the intrinsic cephalosporin resistance of enterococci and serves as new target for anti-enterococcal therapeutics.
\end{abstract}

Though typically benign colonizers of the gastrointestinal tracts of a diverse array of organisms, enterococci are also important nosocomial pathogens. In the years 2009-2010, enterococci accounted for $13.9 \%$ of healthcare-associated infections (HAIs) ${ }^{1}$. While this prevalence alone underscores the importance of enterococci as pathogens, it is compounded by the organisms' well-known possession of both intrinsic and acquired resistance determinants to a number of antibiotics, some of which can be transferred to other organisms 2,3 . Particularly problematic are the greater than $80 \%$ of $E$. faecium HAIs and the increasing proportion of $E$. faecalis HAIs that are vancomycin-resistant ${ }^{1,4}$.

One risk factor for enterococcal infection is prior treatment with cephalosporin antibiotics $2,3,5$. Cephalosporins are $\beta$-lactam antibiotics that function by inhibiting the transpeptidase activity of penicillin-binding proteins (PBPs). Diminished or absent transpeptidation weakens the peptidoglycan, resulting in lysis. Enterococcal resistance to cephalosporins is intrinsic; it is encoded in the core genome and is a characteristic of nearly

*Corresponding author: ckristich@mcw.edu.

Supporting information. Supporting information including 3 tables, 4 figures, and associated text accompanies this manuscript. The authors declare no conflicts of interest. 
all isolates $1,6,7$. Even at concentrations above the MIC, $\beta$-lactams, including cephalosporins, are not bactericidal to enterococci ${ }^{8}$.

While progress has been made towards elucidating the mechanism of intrinsic resistance to cephalosporins, our current knowledge is incomplete. The best-characterized factor required for intrinsic cephalosporin resistance of enterococci is the low-affinity penicillin binding protein Pbp5. Because it is not inhibited by high concentrations of cephalosporins, Pbp5 maintains the integrity of the peptidoglycan layer even in their presence ${ }^{9}, 10$. However, Pbp5 alone is not sufficient for resistance. For example, deletion of a prototypical response regulator/ sensor histidine kinase pair, CroRS, impairs intrinsic cephalosporin resistance via an unknown mechanism ${ }^{11}$. Presumably, sensitivity to cephalosporins results from the loss of proper gene regulation in Cro mutants, but as yet, the CroR-dependent genes important for cephalosporin resistance remain to be identified. Similarly, deletion of the serine/ threonine kinase IreK dramatically sensitizes $E$. faecalis to cephalosporin antibiotics ${ }^{12}$. Although the kinase is required, downstream factors in the IreK signaling pathway that are critical for cephalosporin resistance have not been established. Hence, additional factors that influence cephalosporin resistance in $E$. faecalis must remain to be identified.

To help identify such factors, we sought small molecules capable of potentiating cephalosporin activity towards E. faecalis. Screens to identify potentiators have identified new factors critical for cell-wall synthesis that may themselves be targets for new antibiotics ${ }^{13}$. Additionally, potentiators of $\beta$-lactam antibiotics show promise as therapeutic adjuvants to overcome $\beta$-lactam-resistant Gram-positive bacteria ${ }^{13}, 14$. Here we describe follow-up studies of a subset of the identified molecules - specifically those that inhibit dihydrofolate reductase (DHFR, EC 1.5.1.3) or thymidylate synthase (TS, EC 2.1.1.45). Inhibition of DHFR prevents synthesis of tetrahydrofolate and subsequently $\mathrm{N}^{5}, \mathrm{~N}^{10}$ methylene-tetrahydrofolate, the methyl donor for TS, thus both compounds ultimately disrupt thymidylate synthesis. We demonstrate that thymidine limitation influences cephalosporin resistance by restricting available dTDP-loaded precursors necessary for biosynthesis of cell-wall polysaccharide. These studies therefore identify a new determinant of cephalosporin resistance in enterococci.

\section{Results and Discussion}

\section{Identification of compounds that potentiate cephalosporin activity}

Over 74000 compounds were screened for their ability to potentiate the activity of ceftriaxone (a broad-spectrum cephalosporin, CTRX) against wild-type E. faecalis OG1RF (Figure S1). Approximately 1700 compounds (2.3\% hit rate) reduced total ATP upon cotreatment with CTRX as compared to treatment with CTRX alone, of which approximately 1550 were subsequently screened in both the presence and absence of CTRX. Compounds with potent anti-enterococcal activity in the absence of CTRX were removed from further analysis. Ultimately, 260 of the 1550 molecules were selected for dose-response analysis, after which approximately 75 compounds met our final inclusion criteria: a pIC50 $\geq 3$ in the presence of CTRX that exceeded the pIC50 value in its absence by a minimum of one unit (pIC50 is the negative $\log$ of the concentration required to get 50\% inhibition [IC50]; a higher pIC50 represents a more potent compound). 


\section{MWP01114 and MWP01127 inhibit DHFR}

Few of the active compounds identified in the high throughput screen had known targets. However, 5'-fluorouracil (5FU), which directly inhibits thymidylate synthase (TS), and pyrimethamine, which inhibits dihydrofolate reductase (DHFR), were among the active compounds. The active compounds MWP01114 [5-(4-fluorophenoxy)quinazoline-2,4diamine, pIC50=5.24] and MWP01127 [5-(4-methylphenyl)sulfanylquinazoline-2,4diamine, pIC50=5.6] each contain a diaminopyrimidine ring (Figure 1), as do pyrimethamine and trimethoprim (TMP), suggesting that these newly identified active compounds were also DHFR inhibitors. To test their activity, we determined minimum inhibitory concentrations (MIC) when DHFR was expressed in trans from a multi-copy plasmid. Expression of DHFR in wild-type E. faecalis increased the MIC of MWP01114 and MWP01127 to the same extent as seen with pyrimethamine and TMP (Table S1). Similarly, expression of TS increased the MIC for 5FU compared to empty vector (Table S1). Although the changes in MIC for the active compounds are modest, they are specific and mimic the changes observed with known DHFR inhibitors, indicating that MWP01114 and MWP01127 inhibit DHFR. Therefore, we used TMP as a model DHFR inhibitor for the following experiments.

\section{Inhibition of DHFR acts synergistically with cephalosporins against $E$. faecalis}

Because TMP and 5FU possess inherent antimicrobial activity against enterococci, we employed checkerboard analyses to probe for evidence of synergy with cephalosporins. We determined the fractional inhibitory concentration (FIC) index of each combination as described ${ }^{15}$. FIC indices $\leq 0.5$ are widely considered indicative of synergy between 2 compounds ${ }^{15}$, however we chose a more stringent definition of $<0.5$. By this criterion both TMP plus CTRX, and 5FU plus CTRX exhibited synergy (Table 1), with several sub-MIC combinations resulting in an FIC index $<0.5$ (Figure S2). This confirmed that inhibition of DHFR or TS acts synergistically with CTRX against $E$. faecalis in vitro. We also tested TMP or 5FU with ceftazidime (another broad-spectrum cephalosporin), cefuroxime (an expandedspectrum cephalosporin), and cefadroxil (a narrow-spectrum cephalosporin). Combinations including expanded and broad-spectrum cephalosporins demonstrated synergy, while combinations including cefadroxil did not (Table 1). Other $\beta$-lactams proved more variable: ampicillin and ertapenem exhibited synergy in combination with 5FU, but not with TMP, and imipenem was not synergistic with TMP or 5FU (Table 1). Together, these results suggest that the synergistic activity of thymidylate limitation is generalizable to expanded and broad-spectrum cephalosporins, though not to all $\beta$-lactams.

Previous studies have described synergy between cell-wall active antibiotics and aminoglycosides towards $E$. faecalis isolates with low to moderate-level aminoglycoside resistance. The prevailing model for this phenomenon is that the cell-wall active agent weakens the cell-wall, increasing access of the aminoglycoside to its intracellular target 16,17 . To test the role of this mechanism in the synergy exhibited between TMP and CTRX we performed two complementary sets of experiments. First, we tested other cellwall targeting antibiotics in combination with TMP or 5FU. While a few combinations exhibited synergy towards E. faecalis, the FIC indices were closer to 0.5 than those obtained with CTRX (Table 1). Moreover, the effect - where observed - was not common to both 
TMP and 5FU. In the second experiment, we tested whether CTRX exhibited synergy with antibiotics that inhibit intracellular processes other than thymidylate production. None of the antibiotics tested in combination with CTRX met our strict definition of synergy. Based on these data, and the lack of synergy between a narrow-spectrum cephalosporin and TMP or $5 \mathrm{FU}$, we conclude that this effect is not solely the result of increased accessibility of intracellular targets due to a weakened cell wall.

To examine the specificity of TMP or 5FU for cephalosporins, we also tested for synergy between TMP or 5FU and antibiotics with non-cell-wall targets. Only 1 instance of moderate synergy was observed (TMP with chloramphenicol) Table 1). Because no synergy was observed between 5FU and chloramphenicol, it is unlikely that the synergy observed between TMP and chloramphenicol is due to thymine limitation.

It is well-established that $E$. faecalis can import and utilize exogenously provided thymine or folate analogs, circumventing the need for functional DHFR or TS ${ }^{18-20}$. Therefore, we tested whether providing exogenous thymine would antagonize the synergy exhibited by TMP or 5FU in combination with CTRX. Indeed, when provided in the culture medium, supplementary thymine prevented determination of an inhibitory concentration of TMP or 5FU, and neither compound was able to potentiate the activity of CTRX (the MIC for CTRX was unchanged from medium lacking TMP or 5FU). This strengthens the conclusion that reduced thymidylate production, rather than other potential consequences of DHFR or TS inhibition, impairs cephalosporin resistance of $E$. faecalis. Because thymine is presumably available to $E$. faecalis in the context of human infections inferred from failure of TMP therapy for enterococcal infections ${ }^{21,22}$ ), these results also illustrate that DHFR or TS inhibitors are unlikely to be successful therapeutic adjuvants during cephalosporin therapy. However, they suggest the presence of factors downstream of thymidylate production that contribute to intrinsic cephalosporin resistance. Such factors may therefore represent novel therapeutic targets.

We next asked if co-treatment with TMP and CTRX resulted in bacterial killing or simply prevented growth. To mimic conditions of the checkerboard analysis, wild-type E. faecalis OG1RF was grown to stationary phase and then sub-cultured in the presence of TMP, CTRX, or both. At the concentrations tested, neither TMP nor CTRX prevented growth of the bacteria (Figure 2). However, TMP combined with CTRX reduced the number of viable cells by approximately $2 \operatorname{logs}$ over the course of the experiment (Figure 2). This demonstrates that the combination of TMP and CFTX can actively kill E. faecalis, which is a highly desirable property for new anti-enterococcal therapeutic strategies.

\section{Determination of species specificity}

To examine if synergy between TMP and CTRX is broadly conserved among enterococci, the combination was tested against distinct lineages of $E$. faecalis, in addition to Enterococcus faecium, Enterococcus casseliflavus, and Enterococcus gallinarum. TMP and CTRX exhibited synergy against all strains of $E$. faecalis and $E$. faecium tested (Table 2), however, we did not detect synergy against $E$. casseliflavus, E. gallinarum, or the methicillin-resistant Staphylococcus aureus strain MW2. Thus, the enhanced antibiotic 
activity of CTRX in the presence of TMP is applicable to the most clinically relevant enterococci, E. faecalis and E. faecium, though not to all Gram-positive organisms.

\section{Mutants impaired at dTDP-glucose synthesis are more sensitive to ceftriaxone}

Starvation for thymine is expected to interfere with DNA replication. Our initial hypothesis that an increase in mutation frequency mediated the synergy between TMP and CTRX was not supported experimentally (see Supplemental Information, Figure S3). However, dTTP is necessary to generate dTDP-glucose, a biosynthetic precursor of dTDP-rhamnose, which is subsequently used for exopolysaccharide production in many bacteria. E. faecalis possesses the machinery to convert dTDP-glucose into dTDP-rhamnose, and we predict it to be functional because rhamnose is a constituent of the 37-50 kDa cell-wall polysaccharide, Epa ${ }^{23-26}$. We hypothesized that restriction of available thymidylate due to TMP or 5FU treatment impairs Epa production, thereby causing changes in the cell-wall that result in synergy between these drugs and cephalosporins. In E. faecalis OG1RF, dTDP-rhamnose production is predicted to be catalyzed by the gene products of $O G 1 R F_{-} 11734-$ $O G 1 R F_{-} 11731$, also called epaE- $H^{26}$. Mutations in the epa gene cluster lead to dramatic changes in the Epa polysaccharide ${ }^{25-28}$ and result in reductions in biofilm formation, immune evasion, conjugation, and pathogenicity ${ }^{25-29}$ in addition to changes in cell morphology 26,28 . For consistency with the diverse range of organisms that encode orthologous dTDP-rhamnose biosynthesis machinery, we will employ the naming scheme proposed by Reeves, et al ${ }^{30}$ and refer to $O G 1 R F_{-} 11734$ as $r m l A$.

Because RmlA (glucose-1-phosphate thymidyltransferase, EC 2.7.7.24) explicitly requires dTTP as a substrate, we reasoned that if the mechanism of synergy between TMP and CTRX is indeed through impaired exopolysaccharide production, inactivation of $r m I A$ would similarly result in increased sensitivity to cephalosporins (in the absence of TMP). In-frame deletion of $r m I A$ from wild-type $E$. faecalis OG1 resulted in a severe growth defect, marked by reduced maximal density and increased autolysis (not shown), unlike what was previously reported for an $r m l A$ insertion mutant ${ }^{26,29}$. However, in previous reports the insertional inactivation of $r m l A$ exerted polar effects on the expression of downstream genes, which may account for this discrepancy ${ }^{26,29}$. Inconsistencies in growth kinetics among multiple independent isolates of the $E$. faecalis $\triangle \mathrm{rml} A$ strain suggested suppressor mutations enabling improved growth were rapidly acquired (not shown). To circumvent this obstacle, we attempted to create a temperature-sensitive allele of $r m l A$ by making substitutions at buried residues as previously described ${ }^{31,32}$. We selected 2 variants (encoding $\mathrm{RmIA}^{\mathrm{I} 5 \mathrm{H}}$ and $\mathrm{RmlA}^{\mathrm{I5W}}$ ) that permitted wild-type growth on agar plates at permissive temperature $\left(30^{\circ} \mathrm{C}\right)$, but mimicked the growth defect of the $\Delta r m I A$ strain at non-permissive temperature $42^{\circ} \mathrm{C}$ ) (Table S3), and introduced them into the native copy of $r m I A$ in the E. faecalis chromosome. Growth of the resulting mutants at non-permissive temperature was substantially impaired (Figure 3a, Figure S4), mimicking the dramatic growth defect of the $\triangle r m l A$ mutant. Moreover, the mutants exhibited a substantial loss of ceftriaxone resistance at non-permissive temperature (Table 3), consistent with our model that interfering with Epa exopolysaccharide production by limiting dTDP-rhamnose production sensitizes E. faecalis to cephalosporins. 
Surprisingly, at permissive temperature, both mutants exhibited a modest increase in cephalosporin resistance (Table 3), and a moderate growth defect in liquid media (Figure 3, Figure S4). Therefore, it seems likely that the RmlA variants tested are not fully functional at permissive temperature. The altered resistance seen at permissive temperature is consistent in general with a role for the Epa polymer in cephalosporin resistance, though it is unclear why perturbation of RmlA at $30^{\circ} \mathrm{C}$ results in increased - rather than decreased resistance. The dynamics of cell-wall homeostasis are likely altered during slower growth at the lower temperature, which might allow the cell to compensate for defects in the Epa polymer via another mechanism that leads to modestly enhanced cephalosporin resistance.

\section{Examination of exopolysaccharide}

Lesions in the epa locus, which includes $r m I A$, alter the properties of the enterococcal Epa exopolysaccharide ${ }^{25-28}$. To visually examine the effects of the $\mathrm{RmlA}^{\mathrm{I5H}}$ and $\mathrm{RmlA}^{\mathrm{I}} \mathrm{W}$ mutations on the Epa polymer, we extracted exopolysaccharide from wild-type E. faecalis, $\triangle r m l A, \mathrm{RmlA}^{\mathrm{I5H}}$, and $\mathrm{RmlA}^{\mathrm{ISW}}$ mutants at permissive and non-permissive temperatures. We reasoned that any suppressors that accumulated during growth of the $\triangle r m l A$ mutant would be unlikely to re-enable the synthesis of dTDP-glucose (due to absence of $r m I A$ ), allowing us to use $\triangle r m l A$ mutant extracts as a control to visualize the influence of RmlA on exopolysaccharide. Fifty micrograms of exopolysaccharide were separated on a $10 \%$ polyacrylamide gel and visualized using the cationic dye Stains-All. A dramatic shift in migration, consistent with previous reports ${ }^{26}$, was observed when comparing extracts from the $\mathrm{RmlA}^{\mathrm{I}}{ }^{\mathrm{H}}$ and $\mathrm{RmlA}^{\mathrm{I5W}}$ mutants at non-permissive temperature to those from wild-type bacteria Figure $3 \mathrm{~b}$ ). The shift mimicked that seen when comparing polysaccharide from the $\triangle r m l A$ mutant to the wild-type, confirming that $\mathrm{RmlA}^{\mathrm{ISH}}$ and $\mathrm{Rml}^{\mathrm{I}}{ }^{\mathrm{WW}}$ are non-functional at non-permissive temperature. Furthermore, at permissive temperature, the $15 \mathrm{H}$ mutant exhibited two species of polymer - one that migrated similarly to wild-type polysaccharide and another that migrated with exopolysaccharide from the $\Delta r m l A$ mutant. At nonpermissive temperature the slower species disappeared (Figure $3 b$ ). We infer that $\mathrm{RmlA}^{\mathrm{I}} \mathrm{H}$ is sufficiently functional at permissive temperature to enable production of some wild-type Epa polymer, which vanishes upon inactivation of $\mathrm{RmlA}^{\mathrm{I5H}}$ at non-permissive temperature. These results are consistent with our previous conclusion that the RmlA temperaturesensitive mutants are somewhat impaired at permissive temperature.

These results suggest that thymidylate-limitation may be an effective antagonist of exopolysaccharide production in organisms that require a dTDP-glucose intermediate, i.e., those organisms that encode an RmlA homolog. Drugs targeting the Rml pathway are currently in development for pathogens such as Mycobacterium tuberculosis ${ }^{33,34}$. We speculate that thymidylate limitation could act synergistically with such inhibitors of the Rml pathway.

\section{Cell-wall integrity analysis of $r m I A$ mutants}

Although E. faecalis mutants with lesions in the epa gene cluster exhibit a variety of phenotypes, a precise molecular function for the Epa polymer has not been established. Given the reduced resistance of RmlA mutants to the cell-wall-active antibiotic CTRX, we hypothesized that cell-wall integrity was compromised by rmlA mutations. To test this, we 
used a modified small molecule permeability assay based on chlorophenol redgalactopyranoside $(\mathrm{CPRG})^{35,36}$. CPRG is normally excluded from wild-type E. faecalis cells; however, mutations that result in cell-envelope defects permit CPRG entry. Hydrolysis by intracellular $\beta$-galactosidase then releases a visible and quantifiable red product ${ }^{36}$. Wildtype and mutant $E$. faecalis constitutively expressing $\beta$-galactosidase were incubated at high density at either permissive or non-permissive temperature in media containing CPRG. Bacterial cell density of all strains increased 2-fold over the first 2 hours, after which it remained constant. At permissive temperature, there was no difference in CPRG cleavage between the wild-type and $\mathrm{RmlA}^{\mathrm{I} 5 \mathrm{H}}$ or $\mathrm{RmlA}^{\mathrm{I} 5 \mathrm{~W}}$ mutants (Figure 3c); however, after 6 hours at non-permissive temperature, the mutants exhibited significantly higher amounts of CPRG hydrolysis compared to the otherwise isogenic wild-type strain (Figure 3c), indicative of defects in cell-wall integrity as a result of RmlA inactivation.

\section{Inhibiting thymidylate synthesis impairs cell wall integrity}

Given that genetic perturbation of RmlA activity impaired cell-wall integrity, we hypothesized that pharmacologically restricting availability of an RmlA substrate (thymidylate) via treatment with TMP or 5FU should similarly impair wall integrity. Growth of wild-type $E$. faecalis expressing $\beta$-galactosidase was severely inhibited on agar plates containing TMP or $5 \mathrm{FU}$ without thymine; however, where present, colonies were surrounded by a red halo indicative of CPRG hydrolysis. Addition of $1 \mu \mathrm{g} / \mathrm{ml}$ of thymine restored growth but not cell-wall integrity, as CPRG was still robustly hydrolyzed. Additional thymine ( $5 \mu \mathrm{g} / \mathrm{ml}$ ) both restored growth to wild-type levels, and reduced CPRG hydrolysis to the levels seen in the absence of TMP or 5FU (Figure 4). We conclude that an inadequate supply of thymidylate, due to DHFR or TS inhibition, results in diminished cell-wall integrity.

\section{Treatment with trimethoprim reduces the amount of rhamnose in $E$. faecalis exopolysaccharide}

We demonstrated that TMP treatment diminishes cell-wall integrity, and hypothesized this was due to alterations in the Epa polymer. Cell-wall polysaccharide isolated from exponentially growing cells in the presence of sub-inhibitory TMP did not exhibit changes when separated on a polyacrylamide gel (not shown). Based on the results of the RmlA temperature-sensitive mutants (Fig $3 \mathrm{a}$ and $3 \mathrm{~b}$ ), we hypothesized that TMP concentrations required to visualize a change in the Epa exopolysaccharide by electrophoresis would result in considerable growth inhibition, prohibiting production and isolation of polysaccharide. To detect more subtle changes in the Epa polymer, we assessed rhamnose content of extracted exopolysaccharide after treatment with TMP. Because rhamnose is the only methylpentose known to be present in exopolysaccharide of E. faecalis and is uniquely found in the Epa polymer ${ }^{23,25}$, we used the method of Dische and Shettles ${ }^{37}$ for methylpentose quantification. Cell-wall polysaccharide isolated from wild-type $E$. faecalis grown in the presence of sub-inhibitory TMP $(0.0625 \mu \mathrm{g} / \mathrm{ml})$ contained less rhamnose than cell-wall polysaccharide isolated from untreated bacteria $(0.86 \mu \mathrm{g}$ rhamnose/ $10 \mu \mathrm{g}$ dry weight $\mathrm{v} 0.98$ $\mu \mathrm{g}$ rhamnose/ $10 \mu \mathrm{g}$ dry weight, Figure $4 \mathrm{~b})$. 
Our screen to identify potentiators of cephalosporins with activity against enterococci revealed a new factor critical for enterococcal cephalosporin resistance - the Epa exopolysaccharide. Because cephalosporin antibiotics interfere with peptidoglycan synthesis, it is perhaps unsurprising that other disruptions to cell-wall homeostasis, e.g. impaired exopolysaccharide production, are capable of sensitizing otherwise resistant bacteria to cephalosporins. We propose a mechanism by which thymidylate limitation, in addition to its widely studied effects on DNA replication, modulates exopolysaccharide production, thus affecting cell-wall homeostasis (Figure 5). The mechanism behind the disruption of cell-wall homeostasis may be through enzyme localization. For example, in methicillin-resistant $S$. aureus disrupting proper localization of certain PBPs by inhibition of wall-teichoic acid production ${ }^{39-41}$ or of other cell-wall machinery using chemical inhibitors ${ }^{13,14}$ results in sensitization to $\beta$-lactams. In Group B Streptococcus, the Group B antigen (a carbohydrate) is necessary for proper localization of a peptidoglycan hydrolase; inactivation of the first step of carbohydrate synthesis results in dramatic alterations in growth and division ${ }^{38}$. While the ability of $E$. faecalis to avoid inhibition by DHFR inhibitors 21,22 reduces the likelihood of novel enterococcal therapeutics based on these molecules, our results suggest that molecules that target $\mathrm{rmlA}$, or exopolysaccharide production in general, may be effective adjuvants and merit further investigation.

\section{Methods}

\section{Bacterial strains, plasmids and media}

Strains and plasmids are listed in Table S2. E. faecalis was routinely grown in MuellerHinton broth (MHB; Difco). E. coli Top10 and DH5a were used as cloning hosts and grown in half-strength Brain Heart Infusion broth (hBHI; Difco) or Lysogeny Broth (LB). When required, antibiotics were included in the media at the following concentrations: Erythromycin at $10 \mu \mathrm{g} / \mathrm{ml}$ for E. faecalis or $100 \mu \mathrm{g} / \mathrm{ml}$ in hBHI for E. coli; Chloramphenicol at $10 \mu \mathrm{g} / \mathrm{ml}$ for $E$. faecalis and E. coli. Antibiotics and reagents were purchased from Sigma unless otherwise noted.

\section{High-throughput screening}

High-throughput screening was performed at the University of Michigan Center for Chemical Genomics. Wild-type E. faecalis OG1RF was grown overnight in MHB $+10 \%$ Terrific Broth with a final glycerol concentration of $4 \%$. Stationary-phase cultures were diluted into fresh media containing $8 \mu \mathrm{g} / \mathrm{ml} \mathrm{CTRX}$ and incubated overnight at $37^{\circ} \mathrm{C}$ in 384 well plates containing approximately $10 \mu \mathrm{M}$ final concentration of stock compound. Using the BacTiter-Glo reagent (Promega) per manufacturer's instructions, total ATP was measured as a surrogate for cell-density. Confirmation and dose-response screening were performed similarly but included wells lacking CTRX.

\section{Checkerboard Analysis for Synergy determinations}

Checkerboard analysis was performed as described previously, in 96-well plates ${ }^{15}$. Starting inocula were normalized to an $\mathrm{OD}_{600}$ of 0.001 . After $16-18$ hours, $\mathrm{OD}_{600}$ was measured using a SpectraMax M5 (Molecular Devices); an increase in absorbance of at least 0.025 units was considered positive for growth. Rows and columns containing only one antibiotic 
were used to determine the MIC of each compound. The MIC was then used to determine the FIC index. Median values of a minimum of 3 experiments are reported.

Time-kill analysis

Stationary-phase cultures of $E$. faecalis OG1RF were diluted into fresh MHB containing $0.0625 \mu \mathrm{g} / \mathrm{ml} \mathrm{TMP}, 8 \mu \mathrm{g} / \mathrm{ml} \mathrm{CTRX} \mathrm{(GoldBio),} \mathrm{or} \mathrm{both,} \mathrm{and} \mathrm{incubated} \mathrm{at} 37^{\circ} \mathrm{C}$. Starting inocula were normalized to $\mathrm{OD}_{600}$ of 0.001 . Throughout incubation aliquots were removed, washed with water, serially diluted, and plated to hBHI agar. Colonies were enumerated after overnight incubation.

\section{Generation of temperature sensitive $r m / A$ alleles}

To identify buried residues for potential development of temperature-sensitive rmlA mutants we used the method of Varadarjan et al ${ }^{31,32}$. Thirteen different substitutions were made at isoleucine 5, and two at leucine 104 (Table S3). Oligomers were designed to include each desired mutation, and PCR products were introduced into pJRG8 using Gibson Assembly (NEB). Each construct was tested for its ability to complement a $\triangle r m l A$ mutant (JSH119) at $30^{\circ} \mathrm{C}$ and $42^{\circ} \mathrm{C}$ (Table S3). Two mutations (I5H and I5W) were chosen for allelic replacement.

\section{Construction of $r m I A$ mutant strains}

To construct the $\triangle \mathrm{rm} I A$ allele, the 30 terminal nucleotides on each end of the $r m I A$ coding region were amplified with neighboring sequence from OG1 genomic DNA. Amplicons were fused and introduced into pCJK245 ${ }^{42}$ using Gibson Assembly. Plasmids used to generate strains JSH133 and JSH135 were constructed using oligos with the desired mutations to amplify DNA flanking the mutation site, which was then cloned into pCJK245 using Gibson assembly. Plasmids were introduced to E. faecalis OG1 by electroporation, and allelic replacement was performed as described previously 42 .

\section{Determination of antibiotic resistance of JSH133 and JSH135}

Stationary-phase cultures of JSH133 and JSH135 grown in MHB at $30^{\circ} \mathrm{C}$ were diluted to an $\mathrm{OD}_{600}$ of 0.0001 in MHB containing 2-fold dilutions of CTRX. Growth was monitored at $30^{\circ} \mathrm{C}$ and $42^{\circ} \mathrm{C}$ for 24 hours in a Bioscreen C (Oy Biosciences). The MIC was determined as the lowest concentration of CTRX that prevented growth.

\section{Chlorophenol red-galactopyranoside assay for examining cell-wall integrity}

Strains harboring pCJK205, thus constitutively expressing lac $Z$, were concentrated to an $\mathrm{OD}_{600}$ of 0.4 in fresh MHB and incubated at $30^{\circ} \mathrm{C}$ or $42^{\circ} \mathrm{C}$. CPRG was included at 25 $\mu \mathrm{g} / \mathrm{ml}$. $\mathrm{OD}_{570}$ (chlorophenol red absorbance) and $\mathrm{OD}_{630}$ (cell density) were measured at two-hour intervals. One doubling occurred within the first two hours for all strains after which cell density remained constant. The ratio of $\mathrm{OD}_{570} / \mathrm{OD}_{630}$ is reported to indicate normalized CPRG cleavage.

For solid media assays, M9 salts were supplemented with $0.25 \%$ yeast extract, $0.2 \%$ glucose, $1.5 \%$ Bacto agar and $50 \mu \mathrm{g} / \mathrm{ml}$ CPRG. TMP $(1 \mu \mathrm{g} / \mathrm{ml}), 5 \mathrm{FU}(1 \mu \mathrm{M})$ and thymine $(0$, 
1 or $5 \mu \mathrm{g} / \mathrm{ml}$ ) were included as indicated. Ten-fold serial dilutions of wild-type bacteria containing pCJK205 were inoculated on plates for incubation at $37^{\circ} \mathrm{C}$ overnight.

\section{Polyacrylamide analysis of extracted polysaccharide}

Polysaccharide was extracted from exponential cultures of $E$. faecalis grown at $30^{\circ} \mathrm{C}$ or $42^{\circ} \mathrm{C}$ as previously described ${ }^{43}$ with few modifications: bacteria were grown in $\mathrm{MHB}+1 \%$ glucose, and proteinase K (Invitrogen) replaced Pronase. Dry weights of the extracted pellets were determined, and pellets were resuspended at $5 \mathrm{mg} / \mathrm{ml}$. Fifty micrograms of polysaccharide were separated on 10\% TBE-polyacrylamide (33:1) gels at $150 \mathrm{~V}$ and stained with Stains-All in 50\% EtOH in the dark. Destaining was done in water.

\section{Quantification of rhamnose in polysaccharide extracts}

To quantify rhamnose present in extracted polysaccharide we modified the protocol of Dische and Shettles ${ }^{37}$ as follows: $100 \mu \mathrm{l}$ of a $100 \mu \mathrm{g} / \mathrm{ml}$ solution of polysaccharide was mixed with $450 \mu \mathrm{l}$ of $6 \mathrm{H}_{2} \mathrm{SO}_{4}: 1 \mathrm{H}_{2} \mathrm{O}$ on ice. After warming to room temperature, $275 \mu \mathrm{l}$ was removed and boiled for 3 minutes. After cooling in tap water, $110 \mu \mathrm{l}$ was mixed with 2 $\mu \mathrm{l}$ of $0.3 \%$ cysteine- $\mathrm{HCl}$ in a 96 -well plate. Color was allowed to develop for a minimum of 2 hours with shaking. Absorbance was determined at $2 \mathrm{~nm}$ intervals from $360-460 \mathrm{~nm}$ in a SpectraMax M5, and the absorbance of wells lacking cysteine was subtracted. Pure glucose was included to determine the appropriate wavelengths for calculations. Pure rhamnose was included to generate a standard curve. Reported rhamnose content is the mean of 5 independent extractions. Comparisons were done using the Mann-Whitney $\mathrm{U}$ test and GraphPad Prism software. The OG1 $\triangle r m l A$ strain was used to validate this method, and generated no signal as expected.

\section{Supplementary Material}

Refer to Web version on PubMed Central for supplementary material.

\section{Acknowledgments}

This work was supported by grants R01 AI081692 and R21 AI109198 from NIH. The content is solely the responsibility of the authors and does not necessarily represent the official views of the NIH. The funders had no role in study design, data collection and interpretation, or the decision to submit the work for publication. We thank Patrick Schlievert for providing S. aureus MW2 and Michael Gilmore for providing strains of enterococci.

\section{References}

1. Sievert DM, Ricks P, Edwards JR, Schneider A, Patel J, Srinivasan A, Kallen A, Limbago B, Fridkin S. National Healthcare Safety Network (NHSN) Team and Participating NHSN Facilities. Antimicrobial-resistant pathogens associated with healthcare-associated infections: summary of data reported to the National Healthcare Safety Network at the Centers for Disease Control and Prevention, 2009-2010. Infect Control Hosp Epidemiol. 2013; 34:1-14. [PubMed: 23221186]

2. Weigel LM, Clewell DB, Gill SR, Clark NC, McDougal LK, Flannagan SE, Kolonay JF, Shetty J, Killgore GE, Tenover FC. Genetic analysis of a high-level vancomycin-resistant isolate of Staphylococcus aureus. Science. 2003; 302:1569-1571. [PubMed: 14645850]

3. Jasni AS, Mullany P, Hussain H, Roberts AP. Demonstration of conjugative transposon (Tn5397)mediated horizontal gene transfer between Clostridium difficile and Enterococcus faecalis. Antimicrob Agents Chemother. 2010; 54:4924-4926. [PubMed: 20713671] 
4. Hidron AI, Edwards JR, Patel J, Horan TC, Sievert DM, Pollock DA, Fridkin SK. National Healthcare Safety Network Team, Participating National Healthcare Safety Network Facilities. NHSN annual update: antimicrobial-resistant pathogens associated with healthcare-associated infections: annual summary of data reported to the National Healthcare Safety Network at the Centers for Disease Control and Prevention, 2006-2007. Infect Control Hosp Epidemiol. 2008; 29:996-1011. [PubMed: 18947320]

5. Shepard BD, Gilmore MS. Antibiotic-resistant enterococci: the mechanisms and dynamics of drug introduction and resistance. Microbes Infect. 2002; 4:215-224. [PubMed: 11880055]

6. Hamilton-Miller JM. Comparative activity of ampicillin and seven cephalosporins against group D streptococci. J. Clin. Pathol. 1974; 27:828-831. [PubMed: 4214839]

7. Thornton GF, Andriole VT. Laboratory and clinical studies of a new antibiotic, cephaloridine, in the treatment of gram-positive infections. Yale J Biol Med. 1966; 39:9-20. [PubMed: 4382527]

8. Krogstad DJ, Pargwette AR. Defective killing of enterococci: a common property of antimicrobial agents acting on the cell wall. Antimicrob Agents Chemother. 1980; 17:965-968. [PubMed: 6902640]

9. Signoretto C, Boaretti M, Canepari P. Peptidoglycan synthesis by Enterococcus faecalis penicillin binding protein 5. Arch Microbiol. 1998; 170:185-190. [PubMed: 9683658]

10. Arbeloa A, Segal H, Hugonnet J-E, Josseaume N, Dubost L, Brouard J-P, Gutmann L, MenginLecreulx D, Arthur M. Role of class A penicillin-binding proteins in PBP5-mediated beta-lactam resistance in Enterococcus faecalis. J Bacteriol. 2004; 186:1221-1228. [PubMed: 14973044]

11. Comenge Y, Quintiliani R, Li L, Dubost L, Brouard J-P, Hugonnet J-E, Arthur M. The CroRS twocomponent regulatory system is required for intrinsic beta-lactam resistance in Enterococcus faecalis. J Bacteriol. 2003; 185:7184-7192. [PubMed: 14645279]

12. Kristich CJ, Wells CL, Dunny GM. A eukaryotic-type Ser/Thr kinase in Enterococcus faecalis mediates antimicrobial resistance and intestinal persistence. Proc Natl Acad Sci USA. 2007; 104:3508-3513. [PubMed: 17360674]

13. Huber J, Donald RGK, Lee SH, Jarantow LW, Salvatore MJ, Meng X, Painter R, Onishi RH, Occi J, Dorso K, Young K, Park YW, Skwish S, Szymonifka MJ, Waddell TS, Miesel L, Phillips JW, Roemer T. Chemical Genetic Identification of Peptidoglycan Inhibitors Potentiating Carbapenem Activity against Methicillin-Resistant Staphylococcus aureus. Chem Biol. 2009; 16:837-848. [PubMed: 19716474]

14. Tan CM, Therien AG, Lu J, Lee SH, Caron A, Gill CJ, Lebeau-Jacob C, Benton-Perdomo L, Monteiro JM, Pereira PM, Elsen NL, Wu J, Deschamps K, Petcu M, Wong S, Daigneault E, Kramer S, Liang L, Maxwell E, Claveau D, Vaillancourt J, Skorey K, Tam J, Wang H, Meredith TC, Sillaots S, Wang-Jarantow L, Ramtohul Y, Langlois E, Landry F, Reid JC, Parthasarathy G, Sharma S, Baryshnikova A, Lumb KJ, Pinho MG, Soisson SM, Roemer T. Restoring MethicillinResistant Staphylococcus aureus Susceptibility to -Lactam Antibiotics. Science Translational Medicine. 2012; 4:126ra35-126ra35.

15. Pillai, SK.; Moellering, RC., Jr; Eliopoulos, GM. Antimicrobial Combinations. In: Lorian, V., editor. Antibiotics in Laboratory Medicine. 2005.

16. Zimmermann RA, Moellering RC, Weinberg AN. Mechanism of resistance to antibiotic synergism in enterococci. J Bacteriol. 1971; 105:873-879. [PubMed: 4994038]

17. Moellering RC, Weinberg AN. Studies on antibiotic syngerism against enterococci. II. Effect of various antibiotics on the uptake of $14 \mathrm{C}$-labeled streptomycin by enterococci. J. Clin. Invest. 1971; 50:2580-2584. [PubMed: 5001959]

18. Bushby SR, Hitchings GH. Trimethoprim, a sulphonamide potentiator. Br J Pharmacol Chemother. 1968; 33:72-90. [PubMed: 5301731]

19. Zervos MJ, Schaberg DR. Reversal of the in vitro susceptibility of enterococci to trimethoprimsulfamethoxazole by folinic acid. Antimicrob Agents Chemother. 1985; 28:446-448. [PubMed: 3935044]

20. Haltiner RC, Migneault PC, Robertson RG. Incidence of thymidine-dependent enterococci detected on Mueller-Hinton agar with low thymidine content. Antimicrob Agents Chemother. 1980; 18:365-368. [PubMed: 6775593] 
21. Grayson ML, Thauvin-Eliopoulos C, Eliopoulos GM, Yao JD, DeAngelis DV, Walton L, Woolley JL, Moellering RC. Failure of trimethoprim-sulfamethoxazole therapy in experimental enterococcal endocarditis. Antimicrob Agents Chemother. 1990; 34:1792-1794. [PubMed: 2126691]

22. Chenoweth CE, Robinson KA, Schaberg DR. Efficacy of ampicillin versus trimethoprimsulfamethoxazole in a mouse model of lethal enterococcal peritonitis. Antimicrob Agents Chemother. 1990; 34:1800-1802. [PubMed: 2126692]

23. Hancock LE, Gilmore MS. The capsular polysaccharide of Enterococcus faecalis and its relationship to other polysaccharides in the cell wall. Proc Natl Acad Sci USA. 2002; 99:15741579. [PubMed: 11830672]

24. Xu Y, Murray BE, Weinstock GM. A cluster of genes involved in polysaccharide biosynthesis from Enterococcus faecalis OG1RF. Infection and Immunity. 1998; 66:4313-4323. [PubMed: 9712783]

25. Rigottier-Gois L, Madec C, Navickas A, Matos RC, Akary-Lepage E, Mistou M-Y, Serror P. The Surface Rhamnopolysaccharide Epa of Enterococcus faecalis Is a Key Determinant of Intestinal Colonization. J. Infect. Dis. 2014

26. Teng F, Singh KV, Bourgogne A, Zeng J, Murray BE. Further characterization of the epa gene cluster and Epa polysaccharides of Enterococcus faecalis. Infection and Immunity. 2009; 77:37593767. [PubMed: 19581393]

27. Teng F, Jacques-Palaz KD, Weinstock GM, Murray BE. Evidence that the enterococcal polysaccharide antigen gene (epa) cluster is widespread in Enterococcus faecalis and influences resistance to phagocytic killing of $E$. faecalis. Infection and Immunity. 2002; 70:2010-2015. [PubMed: 11895965]

28. Dale JL, Cagnazzo J, Phan CQ, Barnes AMT, Dunny GM. Multiple roles for Enterococcus faecalis glycosyltransferases (GTFs) in biofilm-associated antibiotic resistance, cell envelope integrity, and conjugative transfer. Antimicrob Agents Chemother. 2015

29. Xu Y, Singh KV, Qin X, Murray BE, Weinstock GM. Analysis of a gene cluster of Enterococcus faecalis involved in polysaccharide biosynthesis. Infection and Immunity. 2000; 68:815-823. [PubMed: 10639451]

30. Reeves PR, Hobbs M, Valvano MA, Skurnik M, Whitfield C, Coplin D, Kido N, Klena J, Maskell $\mathrm{D}$, Raetz CRH, Rick PD. Bacterial polysaccharide synthesis and gene nomenclature. Trends in Microbiology. 1996; 4:495-503. [PubMed: 9004408]

31. Varadarajan R, Nagarajaram HA, Ramakrishnan C. A procedure for the prediction of temperaturesensitive mutants of a globular protein based solely on the amino acid sequence. Proc Natl Acad Sci USA. 1996; 93:13908-13913. [PubMed: 8943034]

32. Chakshusmathi G, Mondal K, Lakshmi GS, Singh G, Roy A, Ch RB, Madhusudhanan S, Varadarajan R. Design of temperature-sensitive mutants solely from amino acid sequence. Proc Natl Acad Sci USA. 2004; 101:7925-7930. [PubMed: 15148363]

33. Babaoglu K, Page MA, Jones VC, McNeil MR, Dong C, Naismith JH, Lee RE. Novel inhibitors of an emerging target in Mycobacterium tuberculosis; substituted thiazolidinones as inhibitors of dTDP-rhamnose synthesis. Bioorg. Med. Chem. Lett. 2003; 13:3227-3230. [PubMed: 12951098]

34. Sivendran S, Jones V, Sun D, Wang Y, Grzegorzewicz AE, Scherman MS, Napper AD, McCammon JA, Lee RE, Diamond SL, McNeil M. Identification of triazinoindolbenzimidazolones as nanomolar inhibitors of the Mycobacterium tuberculosis enzyme TDP-6deoxy-d-xylo-4-hexopyranosid-4-ulose 3,5-epimerase (RmlC). Bioorg. Med. Chem. 2010; 18:896-908. [PubMed: 19969466]

35. Paradis-Bleau C, Markovski M, Uehara T, Lupoli TJ, Walker S, Kahne DE, Bernhardt TG. Lipoprotein Cofactors Located in the Outer Membrane Activate Bacterial Cell Wall Polymerases. Cell. 2010; 143:1110-1120. [PubMed: 21183074]

36. Djorić D, Kristich CJ. Oxidative Stress Enhances Cephalosporin Resistance of Enterococcus faecalis through Activation of a Two-Component Signaling System. Antimicrob Agents Chemother. 2015; 59:159-169. [PubMed: 25331701]

37. Dische Z, Shettles LB. A new spectrophotometric test for the detection of methylpentose. J Biol Chem. 1951; 192:579-582. [PubMed: 14907651] 
38. Caliot É, Dramsi S, Chapot-Chartier M-P, Courtin P, Kulakauskas S, Péchoux C, Trieu-Cuot P, Mistou M-Y. Role of the Group B antigen of Streptococcus agalactiae: a peptidoglycan-anchored polysaccharide involved in cell wall biogenesis. PLoS Pathog. 2012; 8:e1002756. [PubMed: 22719253]

39. Farha MA, Leung A, Sewell EW, D’Elia MA, Allison SE, Ejim L, Pereira PM, Pinho MG, Wright GD, Brown ED. Inhibition of WTA Synthesis Blocks the Cooperative Action of PBPs and Sensitizes MRSA to $\beta$-Lactams. ACS Chem Biol. 2013; 8:226-233. [PubMed: 23062620]

40. Campbell J, Singh AK, Santa Maria JP Jr, Kim Y, Brown S, Swoboda JG, Mylonakis E, Wilkinson BJ, Walker S. Synthetic Lethal Compound Combinations Reveal a Fundamental Connection between Wall Teichoic Acid and Peptidoglycan Biosyntheses in Staphylococcus aureus. ACS Chem Biol. 2011; 6:106-116. [PubMed: 20961110]

41. Atilano ML, Pereira PM, Yates J, Reed P, Veiga H, Pinho MG, Filipe SR. Teichoic acids are temporal and spatial regulators of peptidoglycan cross-linking in Staphylococcus aureus. Proceedings of the National Academy of Sciences. 2010; 107:18991-18996.

42. Kristich CJ, Djorić D, Little JL. Genetic basis for vancomycin-enhanced cephalosporin susceptibility in vancomycin-resistant enterococci revealed using counterselection with dominantnegative thymidylate synthase. Antimicrob Agents Chemother. 2014; 58:1556-1564. [PubMed: 24366749]

43. Thurlow LR, Thomas VC, Hancock LE. Capsular polysaccharide production in Enterococcus faecalis and contribution of CpsF to capsule serospecificity. J Bacteriol. 2009; 191:6203-6210. [PubMed: 19684130] 
a)

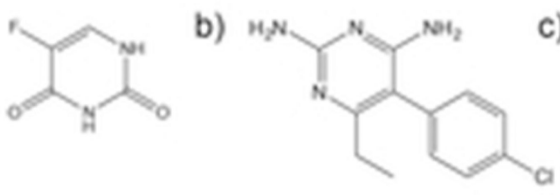

c)

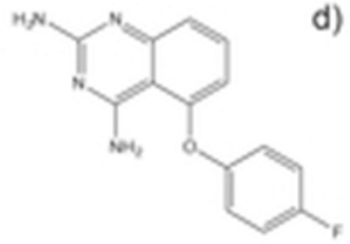

d)

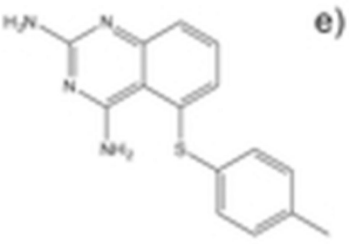

)

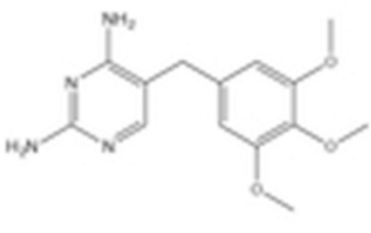

Figure 1. Structures of potentiating compounds

(a) 5'-fluorouracil, (b) pyrimethamine, (c) MWP01114, (d) MWP01127, (e) trimethoprim. 

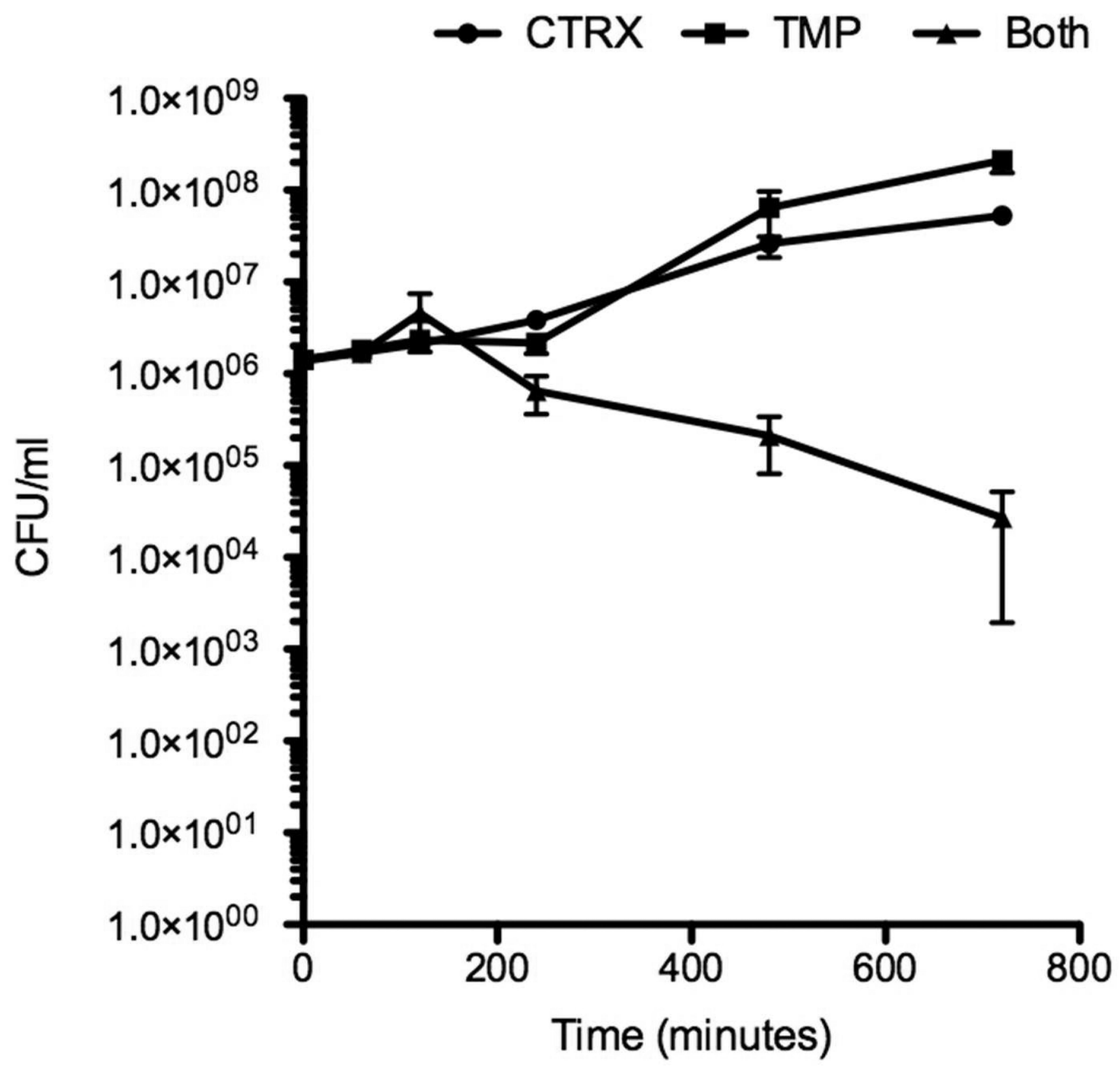

Figure 2. Co-treatment of wild-type $E$. faecalis with TMP and CTRX results in bacterial killing Bacteria were treated with $0.625 \mu \mathrm{g} / \mathrm{ml}$ TMP (squares), $8 \mu \mathrm{g} / \mathrm{ml} \mathrm{CTRX} \mathrm{(circles),} \mathrm{or} \mathrm{both}$ (triangles). Colony forming units (CFU) were enumerated at various times during treatment. Values represent the mean and standard error from 3 independent experiments. 
a)

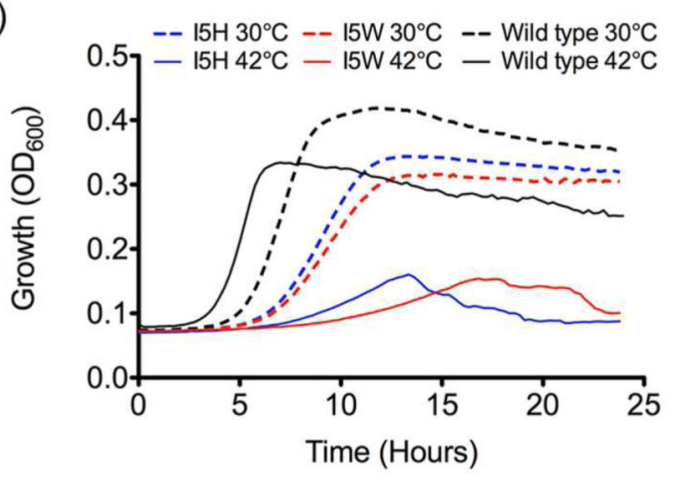

b)

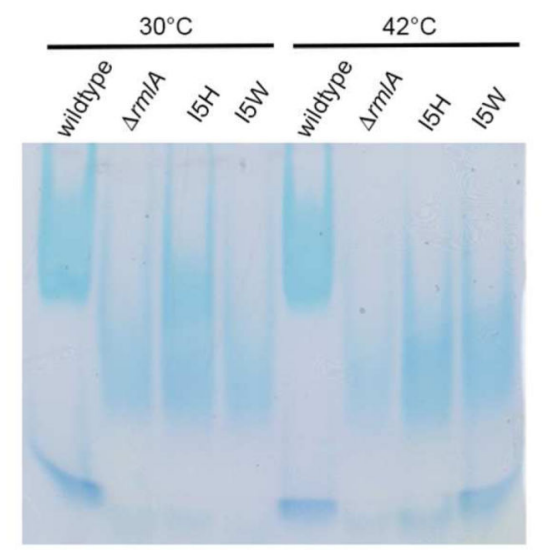

c)

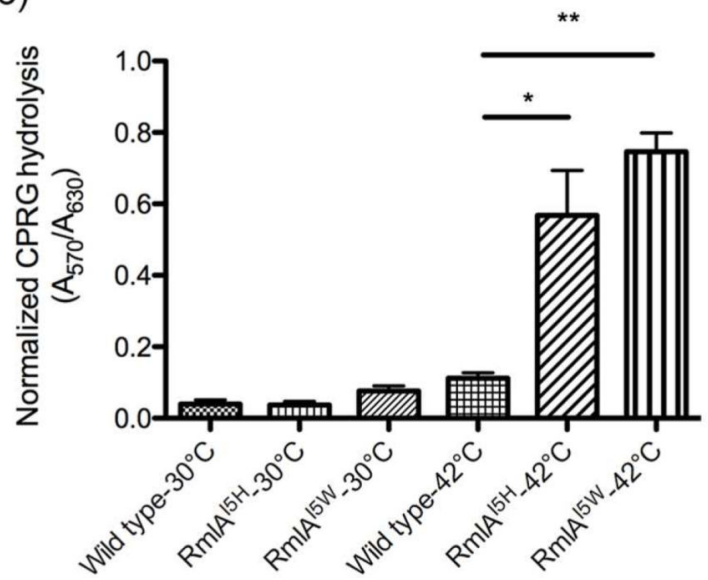

Figure 3. Characterization of $\mathrm{RmIA}^{\mathrm{I5H}}$ and $\mathrm{RmIA}^{\mathrm{I5W}}$ mutants

(a) $\mathrm{RmlA}^{\mathrm{I5H}}$ and $\mathrm{Rml}^{\mathrm{I5W}}$ mutants exhibit a dramatic growth defect at non-permissive temperature. Temperature-sensitive mutants Rml ${ }^{\mathrm{I5H}}$ (JSH133) and RmlA $\mathrm{A}^{\mathrm{I5W}}$ (JSH135), and wild-type $E$. faecalis $\mathrm{OG} 1$, were grown in $\mathrm{MHB}$ at permissive $\left(30^{\circ} \mathrm{C}\right.$; dashed lines) or non-permissive $\left(42^{\circ} \mathrm{C}\right.$; solid lines) temperature for 24 hours in a Bioscreen C. Points represent the mean $\mathrm{OD}_{600}$ from 3-5 independent growth assays. (b) Polysaccharide extracted from $\mathrm{RmlA}^{\mathrm{I5H}}$ and $\mathrm{RmlA}^{\mathrm{I5W}}$ mutants is grossly altered compared to polysaccharide extracted from wild-type E. faecalis. $50 \mu \mathrm{g}$ of polysaccharide extracted from 
wild-type, $\triangle r m l A$ (JSH119), $\mathrm{RmlA}^{\mathrm{I5H}}$, and $\mathrm{RmlA}^{\mathrm{I5W}}$ mutants at $30^{\circ} \mathrm{C}$ and $42^{\circ} \mathrm{C}$ was separated on a $10 \%$ polyacrylamide (33:1) gel and visualized using Stains-All. (c) RmlA ${ }^{15 \mathrm{H}}$ and $\mathrm{RmlA}^{\mathrm{I5W}}$ mutants fail to exclude CPRG at non-permissive temperature. Wild-type $E$. faecalis $\mathrm{OG} 1, \mathrm{Rml}^{\mathrm{I5H}}$, and $\mathrm{Rml}^{\mathrm{I5W}}$ mutants expressing lac $Z$ were incubated in media containing CPRG. After 6 hours CPR accumulation was measured by absorbance at $570 \mathrm{~nm}$ and cell density was measured by absorbance at $630 \mathrm{~nm}$. Normalized CPRG hydrolysis is reported as the ratio of CPR absorbance to cell density. Values represent the mean and standard error of 3 independent experiments. Two-way comparisons were made using t-tests. $* \mathrm{p} \unlhd 0.05, * * \mathrm{p} \quad \unlhd 0.01$. 

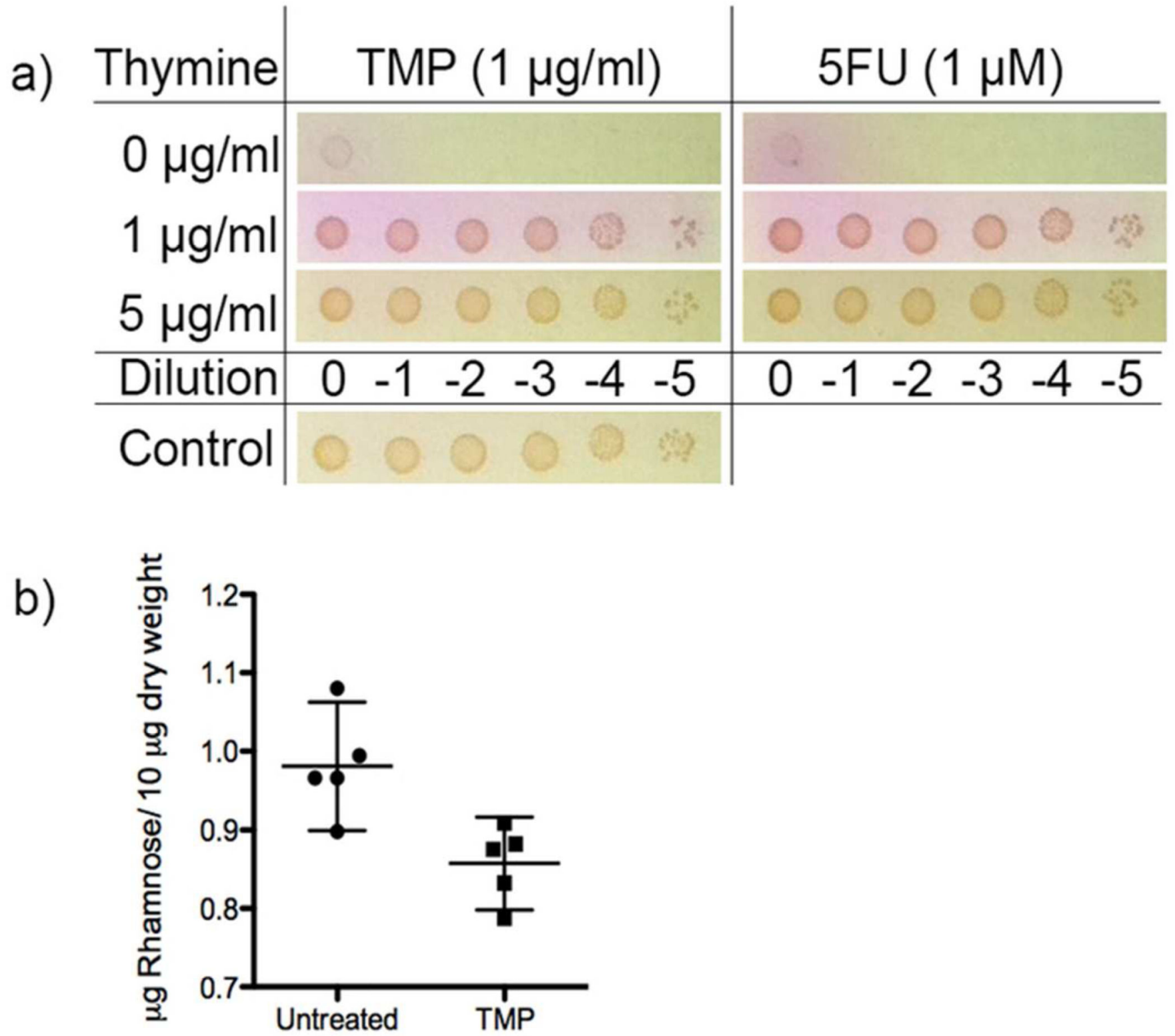

Figure 4. Treatment with TMP or 5FU impairs cell-wall integrity (a) Wild-type E. faecalis OG1 constitutively expressing $l a c Z$ was inoculated on agar plates containing TMP, 5FU, and thymine (Thy) as indicated. Control plates contained no TMP, $5 \mathrm{FU}$, or Thy. CPRG and erythromycin were included in all plates. Log dilutions are indicated. Images are representative after 24 hours of incubation at $37^{\circ} \mathrm{C}$. (b) Polysaccharide was extracted from cultures grown in the absence or presence of sub-inhibitory TMP, and rhamnose content was determined. Mean and $95 \% \mathrm{CI}$ is indicated. $\mathrm{p}=0.02$ using MannWhitney U-test. 
dTDP-

Glucose $-\rightarrow$
dTDP-

Rhamnose"*) Epa

\section{Glucose + dTTP}

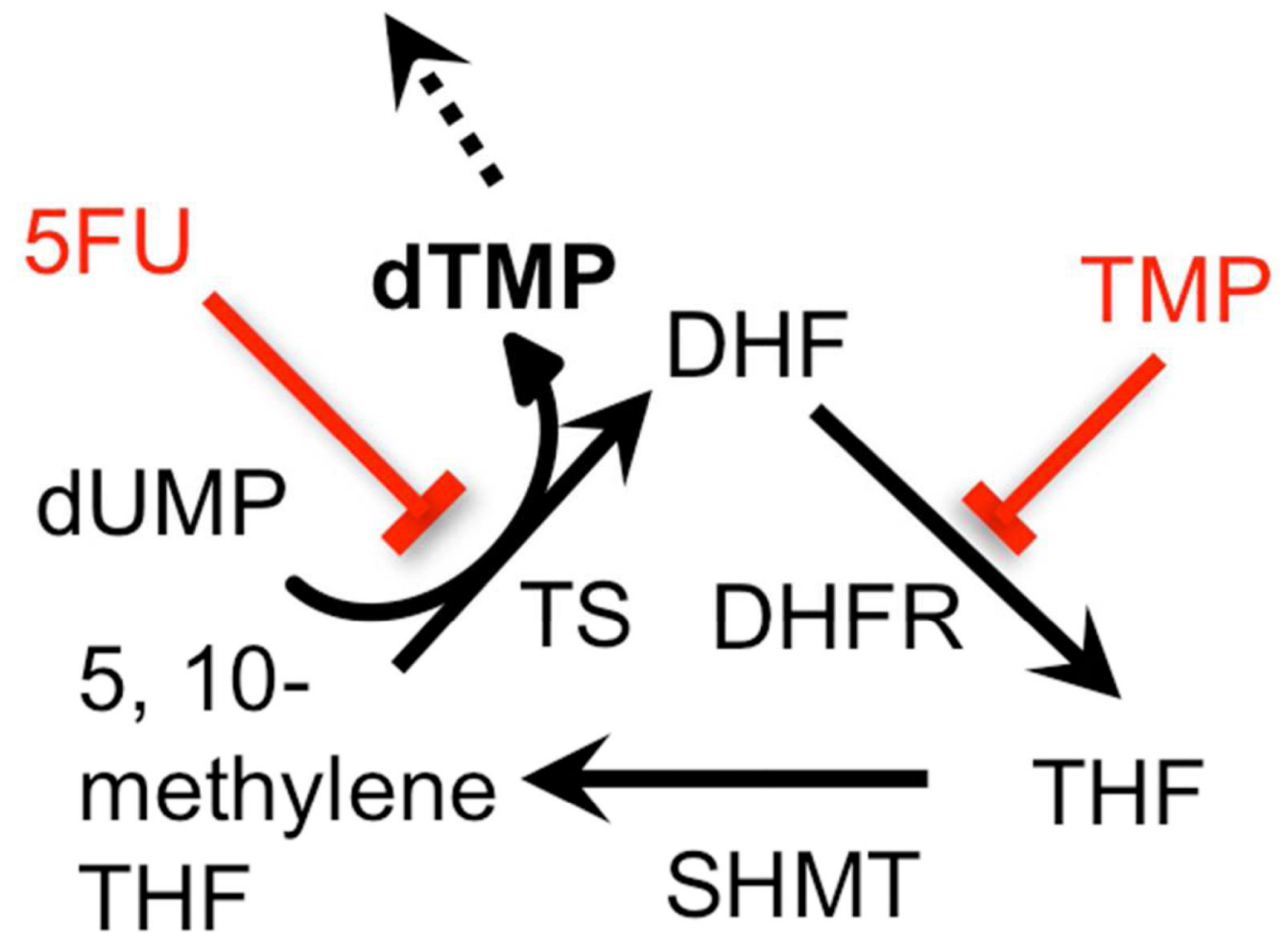

Figure 5. Model for synergy between TMP or 5FU and cephalosporins

TMP and 5FU inhibit steps required for synthesis of dTMP. Reduced levels of dTMP are expected to lower dTTP concentrations, a required substrate for RmlA. Lower amounts of dTDP-glucose result in lower concentrations of dTDP-rhamnose, resulting in sub-optimal Epa production, impairing cell-wall integrity, and increasing sensitivity to cephalosporin antibiotics. Dihydrofolate (DHF), tetrahydrofolate (THF), serine hydroymethyltransferase (SHMT). 


\section{Table 1}

Antimicrobial synergy (denoted in bold) against wild-type E. faecalis OG1RF (values represent median FIC indices [range]).

\begin{tabular}{|c|c|c|c|}
\hline & Trimethoprim & 5-Fluorouracil & Ceftriaxone \\
\hline \multicolumn{4}{|l|}{ Cell Wall } \\
\hline \multicolumn{4}{|l|}{$\beta$-lactam } \\
\hline \multicolumn{4}{|l|}{ Cephalosporin } \\
\hline Ceftriaxone & $\mathbf{0 . 2 5}(0.19-0.38)$ & $\mathbf{0 . 1 9}(0.09-0.19)$ & \\
\hline Ceftazidime & $\mathbf{0 . 3 8}(0.31-0.5)$ & $\mathbf{0 . 2 5}(0.19-0.31)$ & \\
\hline Cefuroxime & $\mathbf{0 . 1 9}(0.12-0.25)$ & $\mathbf{0 . 1 9}(0.19-0.25)$ & \\
\hline Cefadroxil & $0.55(0.53-0.62)$ & $0.55(0.5-0.56)$ & \\
\hline \multicolumn{4}{|l|}{$\underline{\text { Penicillin }}$} \\
\hline Ampicillin & $0.56(0.5-1)$ & $\mathbf{0 . 3 8}(0.38-0.75)$ & \\
\hline \multicolumn{4}{|l|}{$\underline{\text { Carbapenem }}$} \\
\hline Imipenem & $0.5(0.5-0.75)$ & $0.5(0.31-0.75)$ & \\
\hline Ertapenem & $0.5(0.38-0.5)$ & $\mathbf{0 . 3 8}(0.25-0.38)$ & \\
\hline \multicolumn{4}{|l|}{ Other } \\
\hline D-cycloserine & $0.56(0.56-0.63)$ & $0.56(0.56-0.56)$ & \\
\hline Phosphomycin & $\mathbf{0 . 3 1}(0.25-0.5)$ & $0.56(0.31-0.56)$ & \\
\hline Vancomycin & $0.59(0.52-1)$ & $0.69(0.31-0.75)$ & \\
\hline Bacitracin & $0.53(0.5-0.53)$ & $\mathbf{0 . 3 8}(0.38-0.38)$ & \\
\hline \multicolumn{4}{|l|}{ Protein Synthesis } \\
\hline Chloramphenicol & $0.4(0.38-0.5)$ & $0.51(0.28-1)$ & $0.6(0.38-0.63)$ \\
\hline Kanamycin & $0.75(0.51-1)$ & $0.56(0.5-0.75)$ & $0.78(0.51-1)$ \\
\hline Erythromycin & $0.56(0.5-0.56)$ & $0.51(0.29-0.75)$ & $0.53(0.53-0.56)$ \\
\hline \multicolumn{4}{|l|}{ DNA synthesis } \\
\hline Norfloxacin & $0.63(0.56-0.75)$ & $0.75(0.38-1)$ & $0.5(0.38-0.5)$ \\
\hline
\end{tabular}


Table 2

Susceptibility and synergy analysis for other Gram-positive species.

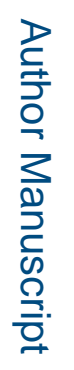

\begin{tabular}{|l|l|l|l|}
\hline Strain & $\begin{array}{l}\text { MIC } \\
\text { Ceftriaxone }(\boldsymbol{\mu g} / \mathbf{m l})\end{array}$ & $\begin{array}{l}\text { MIC } \\
\text { Trimethoprim }(\boldsymbol{\mu g} / \mathbf{m l})\end{array}$ & FIC Index \\
\hline $\begin{array}{l}\text { E. faecalis } \\
\text { (V583) }\end{array}$ & 1024 & 0.125 & 0.27 \\
\hline $\begin{array}{l}\text { E. faecalis } \\
(\mathbf{J H 2})\end{array}$ & 1024 & 2 & 0.17 \\
\hline $\begin{array}{l}\text { E. faecium } \\
(\mathbf{1 , 2 3 1 , 5 0 1 )}\end{array}$ & 1024 & 0.125 & 0.28 \\
\hline $\begin{array}{l}\text { E. faecium } \\
\text { (Com12) }\end{array}$ & 1024 & 0.25 & 0.13 \\
\hline $\begin{array}{l}\text { E. casseliflavus } \\
\text { (EC10) }\end{array}$ & 16 & 0.063 & 0.5 \\
\hline $\begin{array}{l}\text { E. gallinarum } \\
\text { (EG2) }\end{array}$ & 1024 & 0.031 & 1 \\
\hline $\begin{array}{l}\text { S. aureus } \\
\text { (MW2) }\end{array}$ & 32 & 2 & 0.59 \\
\hline
\end{tabular}




\section{Table 3}

Resistance of $\mathrm{Rml}^{\mathrm{I}}{ }^{\mathrm{H}}$ and $\mathrm{Rml}^{\mathrm{I}}{ }^{\mathrm{WW}}$ mutants to ceftriaxone at permissive and non-permissive temperatures. (MICs $(\mu \mathrm{g} / \mathrm{ml})$ are the median of a minimum of three experiments).

\begin{tabular}{llc}
\hline Strain & $\mathbf{3 0}^{\circ} \mathbf{C}$ & $\mathbf{4 2}^{\circ} \mathbf{C}$ \\
\hline Wildtype & 128 & 256 \\
\hline I5H & 1024 & $\leq 4$ \\
\hline I5W & 512 & $\leq 4$ \\
\hline
\end{tabular}

